\title{
Conserving the axilla in breast cancer
}

\author{
Felix Jozsa ${ }^{a}$ (iD) and Muneer Ahmed ${ }^{b}$ iD \\ Division of Surgery and Interventional Science, University College London, Royal Free Hospital, 9th Floor (East), Pond St, London NW3 2QG, UK \\ ahttps://orcid.org/0000-0001-8960-6049
}

bhttps://orcid.org/0000-0002-4504-1354

\section{Abstract}

It is recognised that surgical conservatism is the most effective way of managing the axilla in breast cancer patients undergoing primary breast conserving surgery. The extended clinical scenarios in which a less aggressive approach can be safely adopted warrant consideration-including a group of patients who potentially could bypass surgical staging of the axilla altogether. The application of omission of further surgical management and axillary radiotherapy in the primary surgical and neoadjuvant chemotherapy settings are considered.

Keywords: breast cancer, axillary node metastasis, sentinel node biopsy, primary systemic therapy, axillary node clearance, radiotherapy

\section{Introduction}

Surgical axillary nodal status remains relevant for the determination of adjuvant systemic therapies in breast cancer. Modern breast cancer care is based upon multimodal management and has since evolved several diagnostic and therapeutic techniques that allow for a more nuanced approach with the aim of avoiding complete axillary node clearance (ANC) where possible. This includes genomic assessments, such as MammaPrint [1], Oncotype $\mathrm{dx}$ [2] and Endopredict [3]. Patients may present with nodal disease detectable only on imaging or with clinically palpable disease. The advent of the use of primary systemic therapies (PST) adds further distinct patient groups to be considered. Here, we review the current evidence for surgical and non-surgical management strategies of axillary nodal metastases in breast cancer amongst all these patient subsets.

\section{Patients undergoing primary breast surgery}

For patients presenting with primary invasive breast cancer, current practice with regards to the axilla includes a clinical and radiological assessment (most commonly ultrasound) at the time of work up [4]. The clinical assessment of the nodal status is vitally important as positive and negative groups represent distinct patient populations. The current standard of practice subdivides these two groups into further distinct groups based on the
Correspondence to: Felix Jozsa

Email: felixjozsa@gmail.com

ecancer 2020, 14:1090

https://doi.org/10.3332/ecancer.2020.1090

Published: $18 / 08 / 2020$

Received: 24/04/2020

Publication costs for this article were supported by ecancer (UK Charity number 1176307).

Copyright: (c) the authors; licensee ecancermedicalscience. This is an Open Access article distributed under the terms of the Creative Commons Attribution License (http:// creativecommons.org/licenses/by/3.0), which permits unrestricted use, distribution, and reproduction in any medium, provided the original work is properly cited. 
quantified metastatic axillary burden on staging (up to two nodes and greater than two nodes), however, this may not be as relevant as was once thought.

ANC was once a one-size-fits-all tool to enable locoregional control. The subsequent development of sentinel node biopsy (SNB) meant that the pathological status of some axillary nodes could be assessed prior to the completion of ANC. An important further step forward came from the results of the NSABP B-32 [5] and IBCSG 23-01 [6] trials which demonstrated that there was no statistical difference in overall survival (OS) between patients undergoing SNB alone versus SNB and ANC when the initial SNB was negative or positive for micrometastases, respectively. This began the debate regarding the threshold of nodal involvement at which SNB could be considered safe and sufficient management of the axilla. The value of being able to safely substitute SNB for ANC is based upon significantly improved functional arm outcomes, including reduced pain, numbness and swelling [7].

\section{Low and high burden at sentinel node biopsy}

The landmark ACOSOG Z011 [8] trial designated a threshold of two or fewer macrometastatically involved nodes at SNB at which to observe the effect of withholding ANC. This trial enrolled patients with T1-2 primary breast cancers with no palpable lymphadenopathy undergoing primary breast surgery-in the form of breast conserving surgery and adjuvant radiotherapy and systemic therapy-and found no survival benefit for ANC over SNB alone. These results have shaped opinion and practice over the last decade [9] and in this patient group it is now widely accepted that completion ANC is unnecessary [10] - with SNB being considered as therapeutic for low nodal burden axillary disease. Further international clinical trials are underway to see if these results are fully replicable, and to answer some other pending issues raised by the Z11 trial. It is unclear, for example, what absolute value the threshold of two positive nodes has in determining the oncological risk to the patient: if three positive nodes are excised at SNB, could the patient avoid further axillary surgery? To answer this question, the INSEMA [11] trial will now adapt and expand on the Z11 protocol by including patients with up to three macrometastatically involved axillary lymph nodes at SNB. Furthermore, it will not include patients with axillary micrometastases (37.5\% of patients included in Z11 had micrometastases only), helpfully consolidating the patient group being studied. An added consideration to the change of practice heralded after Z11 come from the results of the AMAROS [12] trial which indicate that in patients with a positive SNB, axillary radiotherapy provides equivalent locoregional control when compared with ANC and with less associated morbidity. Unsurprisingly, only $5 \%$ of patients in the AMAROS trial had three or more macrometastatically involved nodes at SNB-as would be expected in a clinically non-palpable axilla. These results should be interpreted in the context of applying axillary radiotherapy as an alternative to ANC in the-clinically negative, sentinel node positive-low burden axilla.

\section{Radiotherapy}

Radiotherapy itself also has adverse side effects-including lymphoedema-and should be avoided where safely possible. In patients with early breast cancer and favourable biomarkers (using the composite IHC4+C score), the PRIMETIME [13] trial will investigate the ipsilateral breast disease recurrence rates when whole-breast radiotherapy is omitted following breast-conserving surgery and negative SNB. The role of axillary conservatism in low-risk groups will, therefore, need to be balanced and considered alongside the reductive use of radiotherapy. The PRIMETIME trial includes SNB, and the conservative omission of completion axillary node clearance relies upon the patient receiving whole-breast radiotherapy. Both strategies cannot be employed simultaneously and the effect of each strategy on recurrence and survival will, therefore, need to be untangled in future clinical trials.

\section{Preoperative ultrasound}

The Z11 trial did not use axillary ultrasound to stratify patients prior to sentinel node biopsy. Since axillary ultrasound is routinely used is in clinical practice, the relevance of it on quantification of axillary burden is an important consideration. 
In the case of a positive pre-operative AUS, the current standard is to either proceed with ANC or to commence upon PST to downstage the axilla. The practice of 'fast-tracking' positive AUS patients to ANC based on the assumption that, owing to its relatively low sensitivity [14], a positive AUS indicates high nodal burden, leads to gross overtreatment of this patient group. Up to $43 \%$ of patients with a positive pre-operative AUS who went on to have ANC had two or fewer involved nodes at surgery [15], meaning these patients could have been provided the same amount of oncological safety with a sentinel node biopsy. This process is complicated by the widespread use of core needle biopsy or fine-needle aspiration cytology (FNAC) to obtain pathology results pre-operatively. The pathological status of a single node biopsied following visualisation on AUS provides no valuable clinical information for the surgeon deciding how to manage the axilla [16], as it does not provide accurate information on the quantification of axillary burden-which requires sentinel node assessment. In view of this, an approach consisting of SNB enhanced by 'clipping' of the core-biopsy confirmed node-to ensure retrieval at surgery using a targeted axillary dissection (TAD)-in the primary surgical setting would provide this accurate staging assessment. This bespoke approach for primary surgery-supported by adjuvant axillary radiotherapy-is currently being explored within the TAXIS trial [17].

The current standard of practice consists of SNB being performed to confirm the node-negative status of the axilla-in the clinically and radiologically negative axilla. However, despite AUS's relatively low specificity, its sensitivity is far more robust. Indeed, a pooled meta-analysis showed that among 5,139 patients with a negative axillary clinical and ultrasound examination pre-operatively, the negative predictive value of AUS was $95.1 \%$ for low nodal burden [16]. This supports that following a negative clinical and AUS pre-operatively, T1-2 breast cancer patients may be able to safely avoid surgical staging of the axilla altogether. Historical precedent for this approach comes from before the emergence of the concept of SNB, when various trials looked at ANC versus no axillary surgery in clinically node-negative patients, and these did not demonstrate a deleterious effect on overall or disease-free survival [11]. Furthermore, in a 7-year follow up of patients with a clinically negative axilla and a negative SNB there was a mere 1.7\% incidence of axillary disease [18]. However, despite this data, the 2019 St. Gallen expert panel [19] recommends awaiting the full results of the SOUND [20] and INSEMA [11] clinical trials before changing practice to omit SNB in this patient group as both trials have arms that compare sentinel node biopsy with observation. Improving biomolecular marker assessment for identification of the $4 \%$ of patients with high nodal disease who present clinically and radiologically negatively is essential. For example, in patients with T1-2, NO, MO disease and a negative SNB although the locoregional recurrence rates were very low as expected, the ACOSOG Z0010 study found higher rates in younger patients and those with hormone-receptor negative tumours [21].

\section{Patients undergoing mastectomy}

Traditionally, patients undergoing mastectomy-regardless of their axillary node status-have been considered as a separate group in terms of the management of the axilla, owing to the omission of adjuvant radiotherapy in certain patients. Current indications for post-mastectomy radiotherapy (PMRT) include tumour size greater than $5 \mathrm{~cm}$, T4 disease, 4 or more positive axillary lymph nodes and positive or close surgical margins [22]. All of the trials considering the benefit of omitting ANC in patients with low axillary node burden undergoing breast conserving surgery included adjuvant whole-breast radiotherapy as a part of protocol. This technique leads to some irradiation of the lower axillary inlet and may, therefore, contribute to locoregional control in that region. However, multiple studies comparing axillary disease recurrence between patients receiving whole-breast radiation alone and partial breast irradiation-in which, depending on the location of the tumour, the axilla would be far less likely to be irradiated-have shown no difference in axillary disease recurrence [11]. Equally, none of the trials completed to date looking at this issue have included patients undergoing mastectomy. This leaves an open question regarding the oncological feasibility of omitting ANC in some patients undergoing mastectomy. Axillary conservatism could subsequently lead to expansion of the indications for PMRT in order to avoid further surgical intervention to the axilla. The POSNOC [23] and SENOMAC [24] trials will include patients undergoing mastectomy and compare outcomes of axillary management versus adjuvant therapy alone in patients with two or fewer positive axillary nodes. These and future trials looking at including mastectomy cases in a Z11-type protocol, provided adjuvant radiotherapy is given will be key to defining the risk in this group. 


\section{Patients undergoing PST}

The use of neo-adjuvant chemotherapy (NACT) in breast cancer is successfully deployed in downsizing the primary tumour to permit breast conserving surgery, thus avoiding mastectomy. Long term survival outcomes in NACT versus adjuvant chemotherapy have been comparable [25]. In terms of the axilla, the patient's nodal status can change pre- and post-NACT, and the value of SNB in this setting remains.

Three clinical trials have examined the false-negative rate (FNR) of SNB following NACT as previous retrospective series had demonstrated highly variable rates-some so high as to nullify the utility of the procedure in this setting [26]. ACOSOG Z1071 [27] reported an overall FNR of $12.6 \%$ in their patient cohort. Although unacceptably high for the predetermined $12 \%$ safety threshold, they found that when dual tracers were employed this reduced to $10.5 \%$ and, furthermore, that removing three or more nodes at SNB reduced the FNR to $9.1 \%$. This was echoed in the SENTINA trial [28], which reported an overall FNR rate of 14.2\%, which reduced to $7.3 \%$ when only patients in whom three or more nodes had been removed at SNB were considered. The SN FNAC trial [29] found comparable FNR rates of $11.5 \%$ when considering macrometastatic deposits as per the protocols for the Z1071 and SENTINA trials. However, when departing from those trial protocols, they found that mandatory use of immunohistochemistry (IHC) and defining metastases less than 0.2 mm as node-positive reduced the FNR to $8.4 \%$.

In patients with node-positive disease prior to NACT, a biopsy can be performed to confirm pathological status of the node, and a radiological clip placed in the node. This is useful as the pathological response to NACT of the clipped node is used as a surrogate for the overall nodal status of the axilla. Indeed, a further finding from the Z1071 trial was that FNR of SNB in the 203 patients with a radiologically clipped node was $6.8 \%$ when the clipped node was in the SNB specimen [30]. So-called TAD, thus involves SNB with selective resection of the previously marked node using iodine-125 seed localisation placed 1-5 days before surgery [31]. The initial report of the use of this technique by Caudle et al [31] in 191 patients demonstrated an FNR of 2.0\% for TAD versus 10.1\% for SNB alone (although this improvement did not reach statistical significance). The marking axillary lymph nodes with radioactive iodine [32] procedure (involves inserting radioactive seed at the time of biopsy (unlike TAD in which the seed is placed 1-5 days before surgery) without performance of SNB and reported a FNR of 7.0\% in 100 patients and an identification rate of $97 \%$.

These techniques seek to optimise the diagnostic and therapeutic value of an augmented sentinel node biopsy procedure to enable the minimum amount of surgical intervention in the axilla post-NACT. Removing the clipped node, and at least three nodes as a part of SNB is evidence-supported to provide good oncological control while avoiding ANC. If, at TAD, residual disease is found in the biopsy-proven node, then further axillary management is needed-current standard of practice would be to perform completion ANC. However, those with low axillary burden are likely being overtreated with this approach. These patients require assessment in future trials of no further management -in the context of breast conserving surgery post-NACT receiving whole breast radiotherapy-and also axillary radiotherapy. The ongoing Alliance A11202 trial [33] will assess whether axillary radiotherapy can provide equivalent locoregional control when compared to ANC in this patient group.

\section{Conclusions}

The evidence-supported direction of axillary surgical management is of decreasing intervention to the axilla. This is supported in both the primary breast surgery and systemically-treated settings. The future refinements of surgery will be based upon the selection of omission of surgical staging, enhancing the bespoke nature of targeted axillary dissection, determination of the significance of residual disease-after PST-and further assessing what truly represents the threshold for low axillary burden. This will herald an exciting era of reduced morbidity for patients.

\section{Conflict of interest statement}

The authors have no disclosures to make concerning financial or personal relationships that could inappropriately influence their work. 


\section{Funding declaration}

No funding was received for this study.

\section{References}

1. Brandão M, Pondé N, and Piccart-Gebhart M (2019) Mammaprint ${ }^{\mathrm{TM}}$ : a comprehensive review Future Oncol 15(2) 207-224 https://doi. org/10.2217/fon-2018-0221

2. McVeigh TP, Hughes LM, and Miller N, et al (2014) The impact of Oncotype DX testing on breast cancer management and chemotherapy prescribing patterns in a tertiary referral centre Eur J Cancer 50(16) 2763-2770 https://doi.org/10.1016/j.ejca.2014.08.002 PMID: 25240289 PMCID: 4204201

3. Dubsky P, Brase JC, and Jakesz R, et al (2013) The EndoPredict score provides prognostic information on late distant metastases in ER+/HER2- breast cancer patients Br J Cancer 109(12) 2959-2964 https://doi.org/10.1038/bjc.2013.671 PMID: 24157828 PMCID: 3859949

4. NCCN Clinical Practice Guidelines in Oncology (NCCN Guidelines ${ }^{\circledR}$ ) - Breast Cancer Version 2.2019 [NCCN.org] Date accessed: 2/07/19

5. Krag DN, Anderson SJ, and Julian TB, et al (2010) Sentinel-lymph-node resection compared with conventional axillary-lymph-node dissection in clinically node-negative patients with breast cancer: overall survival findings from the NSABP B-32 randomised phase 3 trial Lancet Oncol 11(10) 927-933 https://doi.org/10.1016/S1470-2045(10)70207-2 PMID: 20863759 PMCID: 3041644

6. Galimberti V, Cole BF, and Zurrida S, et al (2013) Axillary dissection versus no axillary dissection in patients with sentinel-node micrometastases (IBCSG 23-01): a phase 3 randomised controlled trial Lancet Oncol 14 297-305 https://doi.org/10.1016/S14702045(13)70035-4 PMID: 23491275 PMCID: 3935346

7. Swenson KK, Nissen MJ, and Ceronsky C, et al (2002) Comparison of side effects between sentinel lymph node and axillary lymph node dissection for breast cancer Ann Surg Oncol 9 745-753 https://doi.org/10.1007/BF02574496 PMID: 12374657

8. Giuliano AE, Hunt KK, and Ballman KV, et al (2011) Axillary dissection vs no axillary dissection in women with invasive breast cancer and sentinel node metastasis: a randomized clinical trial JAMA 305 569-575 https://doi.org/10.1001/jama.2011.90 PMID: 21304082 PMCID: 5389857

9. Caretta-Weyer H, Greenberg CG, and Wilke LG, et al (2013) Impact of the American College of Surgeons Oncology Group (ACOSOG) Z0011 trial on clinical management of the axilla in older breast cancer patients: a SEER-medicare analysis Ann Surg Oncol 20(13) 4145-4152 https://doi.org/10.1245/s10434-013-3193-1 PMID: 23959051 PMCID: 3874252

10. Lyman GH, Temin S, and Edge SB, et al (2014) Sentinel lymph node biopsy for patients with early-stage breast cancer: American Society of Clinical Oncology clinical practice guideline update J Clin Oncol 32 1365-1383 https://doi.org/10.1200/JCO.2013.54.1177 PMID: 24663048

11. Reimer T, Stachs A, and Nekljudova V, et al (2017) Restricted axillary staging in clinically and sonographically node-negative early invasive breast cancer (c/iT1-2) in the context of breast conserving therapy: first results following commencement of the intergroupsentinel-mamma (INSEMA) trial Geburtshilfe Frauenheilkd 77(2) 149-157 https://doi.org/10.1055/s-0042-122853 PMID: 28331237 PMCID: 5357224

12. Donker M, van Tisenhoven G, and Straver ME, et al (2014) Radiotherapy or surgery of the axilla after a positive sentinel node in breast cancer (EORTC 10981-22023 AMAROS): a randomised, multicentre, open-label, phase 3 non-inferiority trial Lancet Oncol [published online Oct 16] https://doi.org/10.1016/S1470-2045(14)70460-7 
13. Kirwan CC, Coles CE, and Bliss J, et al (2016) It's PRIMETIME. postoperative avoidance of radiotherapy: biomarker selection of women at very low risk of local recurrence Clin Oncol 28(9) 594-596 https://doi.org/10.1016/j.clon.2016.06.007

14. Alvarez $\mathrm{S}$, Añorbe $\mathrm{E}$, and Alcorta $\mathrm{P}$, et al (2006) Role of sonography in the diagnosis of axillary lymph node metastases in breast cancer: a systematic review Am J Roentgenol 186(5) 1342-1348 https://doi.org/10.2214/AJR.05.0936

15. Ahmed M, Jozsa F, and Baker R, et al (2017) Meta-analysis of tumour burden in pre-operative axillary ultrasound positive and negative breast cancer patients [published correction appears in Breast Cancer Res Treat. 2017 Nov;166(2):337] Breast Cancer Res Treat 166(2) 329-336 https://doi.org/10.1007/s10549-017-4405-3 PMID: 28755147 PMCID: 5668351

16. Jozsa F, Ahmed M, and Baker R, et al (2019) Is sentinel node biopsy necessary in the radiologically negative axilla in breast cancer? Breast Cancer Res Treat 177(1) 1-4 Epub 2019 May 31 https://doi.org/10.1007/s10549-019-05299-5 PMID: 31152326

17. Henke G, Knauer M, and Ribi K, et al (2018) Tailored axillary surgery with or without axillary lymph node dissection followed by radiotherapy in patients with clinically node-positive breast cancer (TAXIS): study protocol for a multicenter, randomized phase-III trial Trials 19(1) 667 Published 2018 Dec 4 https://doi.org/10.1186/s13063-018-3021-9 PMID: 30514362 PMCID: 6278139

18. Galimberti V, Manika A, and Maisonneuve P (2014) Long-term follow-up of 5262 breast cancer patients with negative sentinel node and no axillary dissection confirms low rate of axillary disease Eur J Surg Oncol 40 1203-1208 https://doi.org/10.1016/j.ejso.2014.07.041 PMID: 25186914

19. Untch M, Thomssen C, and Bauerfeind I, et al (2019) Primary therapy of early breast cancer: evidence, controversies, consensus: spectrum of opinion of german specialists on the 16th St. Gallen International Breast Cancer Conference (Vienna 2019) Geburtshilfe Frauenheilkd 79(6) 591-604 https://doi.org/10.1055/a-0897-6457 PMID: 31217628 PMCID: 6570611

20. Gentilini $O$ and Veronesi U, (2012) Abandoning sentinel lymph node biopsy in early breast cancer? A new trial in progress at the European Institute of Oncology of Milan (SOUND: Sentinel node vs Observation after axillary UltraSouND) Breast 21(5) 678-681 https:// doi.org/10.1016/j.breast.2012.06.013 PMID: 22835916

21. Hunt KK, Ballman KV, and McCall LM, et al (2012) Factors associated with local-regional recurrence after a negative sentinel node dissection: results of the ACOSOG Z0010 trial Ann Surg 256(3) 428-436 https://doi.org/10.1097/SLA.0b013e3182654494 PMID: $\underline{22868365}$ PMCID: $\underline{5345746}$

22. Vilarino-Varela M, Chin YS, and Makris A (2009) Current indications for post-mastectomy radiation Int Semin Surg Oncol 65 Published 2009 Feb 9 https://doi.org/10.1186/1477-7800-6-5 PMID: 19203387 PMCID: 2647547

23. Goyal A, Mann B, and Thompson AM (2018) POSNOC trial management group POSNOC: Positive Sentinel Node-Adjuvant therapy alone versus adjuvant therapy plus clearance or axillary radiotherapy J Clin Oncol 36(15_suppl) TPS600-TPS600 https://doi. org/10.1200/JCO.2018.36.15_suppl.TPS600

24. de Boniface J, Frisell J, and Andersson Y, et al (2017) Survival and axillary recurrence following sentinel node-positive breast cancer without completion axillary lymph node dissection: the randomized controlled SENOMAC trial BMC Cancer 17(1) 379 Published 2017 May 26 https://doi.org/10.1186/s12885-017-3361-y PMID: 28549453 PMCID: 5446737

25. Rastogi P, Anderson SJ, and Bear HD, et al (2008) Preoperative chemotherapy: updates of National Surgical Adjuvant Breast and Bowel Project protocols B-18 and B-27 J Clin Oncol 26 778-785 https://doi.org/10.1200/JCO.2007.15.0235 PMID: 18258986

26. Boileau JF, Poirier B, and Basik M, et al (2015) Sentinel node biopsy after neoadjuvant chemotherapy in biopsy-proven node-positive breast cancer: the SN FNAC study J Clin Oncol 33(3) 258-264 https://doi.org/10.1200/JCO.2014.55.7827

27. Boughey JC, Suman VJ, and Mittendorf EA, et al (2012) The role of sentinel lymph node surgery in patients presenting with node positive breast cancer (TO-T4, N1-N2) who receive neoadjuvant chemotherapy: results from ACOSOG Z1071 trial Cancer Res 72 (24 suppl) 
28. Kuehn T, Bauerfeind I, and Fehm T, et al (2013) Sentinel-lymph-node biopsy in patients with breast cancer before and after neoadjuvant chemotherapy (SENTINA): a prospective, multicentre cohort study Lancet Oncol 14(7) 609-618 https://doi.org/10.1016/S14702045(13)70166-9 PMID: 23683750

29. Boileau JF, Poirier B, and Basik M, et al (2015) Sentinel node biopsy after neoadjuvant chemotherapy in biopsy-proven node-positive breast cancer: the SN FNAC study J Clin Oncol 33(3) 258-264 https://doi.org/10.1200/JCO.2014.55.7827

30. Boughey JC, Ballman KV, and Le-Petross HT, et al (2016) Identification and resection of clipped node decreases the false-negative rate of sentinel lymph node surgery in patients presenting with node-positive breast cancer (t0-t4, n1-n2) who receive neoadjuvant chemotherapy: results from ACOSOG Z1071 (Alliance) Ann Surg 263(4) 802-807 https://doi.org/10.1097/SLA.0000000000001375 PMCID: 4777661

31. Caudle AS, Yang WT, and Krishnamurthy S, et al (2016) Improved axillary evaluation following neoadjuvant therapy for patients with node-positive breast cancer using selective evaluation of clipped nodes: implementation of targeted axillary dissection J Clin Oncol 34 10 1072-1078 https://doi.org/10.1200/JCO.2015.64.0094 PMID: 26811528 PMCID: 4933133

32. Donker M, Straver ME, and Wesseling J, et al (2015) Marking axillary lymph nodes with radioactive iodine seeds for axillary staging after neoadjuvant systemic treatment in breast cancer patients: the MARI procedure Ann Surg 261 378-382 https://doi.org/10.1097/ SLA.0000000000000558

33. https://clinicaltrials.gov/ct2/show/NCT01901094 Date accessed: 05/12/19 